\title{
Application of Intensity Sum Rules to the Atomic Tensors and the Dipole and Rotational Strengths Recently Calculated for NHDT
}

\author{
Allan Rupprecht \\ Division of Physical Chemistry, Arrhenius Laboratory, University of Stockholm, S-106 91 Stockholm, Sweden
}

\begin{abstract}
Rupprecht, A., 1991. Application of Intensity Sum Rules to the Atomic Tensors and the Dipole and Rotational Strengths Recently Calculated for NHDT. - Acta Chem. Scand. 45: 439-443.

IR and VCD intensity sum rules have been applied to the atomic tensors and the dipole and rotational strengths recently obtained by Jalkanen et al. [J. Phys. Chem. 92 (1988) 1781 and J. Chem. Phys. 90 (1989) 3204] for the chiral isotopomer of ammonia, NHDT. These authors calculated the atomic tensors with analytical derivative methods at the SCF level of approximation, and via nuclear shielding tensors at the SCF-RPA (random phase approximation) level of approximation. It is found that the intensity sum rules are fulfilled when origin-independent intensities are considered and when all the terms are calculated from the same set of atomic tensors; the data calculated via nuclear shielding tensors required, however, the inclusion of a translational correction for fulfilment in the IR case. The advantage of these intensity sum rules in the present application is that they offer convenient tests of the correctness of intensity calculations in IR and VCD for each given set of atomic tensors, and that they provide an accumulated effect of the basis set considered. For the largest basis sets there is reasonable agreement between results obtained with the two quantum-mechanical approaches.
\end{abstract}

The intensities of the $t$ th fundamental transitions in infrared (IR) and vibrational circular dichroism (VCD) spectra can be rationalized in terms of the dipole strength, $D_{t}$, and the rotational strength, $R_{t}$, respectively. ${ }^{1}$ Within the harmonic approximation for molecular vibrational motion expressions (1a) and (1b) hold for $D_{t}{ }^{1}$ and $R_{t},{ }^{2-6}$ in which

$D_{t}=\frac{h}{2} \frac{1}{\omega_{t}}\left(\frac{\tilde{\mu} \boldsymbol{\mu}}{\partial Q_{t}}\right)_{0}\left(\frac{\partial \boldsymbol{\mu}}{\partial Q_{t}}\right)_{0}$

$R_{t}=\frac{h}{2}\left(\frac{\partial \tilde{\mu}}{\partial Q_{t}}\right)_{0}\left(\frac{\partial m}{\partial \dot{Q}_{t}}\right)_{0}$

$\boldsymbol{\mu}$ and $\boldsymbol{m}$ are the molecular electric and magnetic dipole moments, respectively, $\dot{Q}_{t}$ is the time derivative of the $t$ th normal vibrational coordinate, and $\omega_{t}$ is the corresponding fundamental frequency in radian $\mathrm{s}^{-1}$. The scalar product is written here as a matrix product with the aid of the symbol $\sim$, signifying transpose. The index ${ }_{0}$ refers to the equilibrium configuration of the molecule.

The derivatives in eqn. (1) can be expressed in terms of atomic polar tensors (APTs), $\mathbf{P}_{X(\mu)}^{(a)}=\left(\partial \boldsymbol{\mu} / \partial \boldsymbol{X}^{(a)}\right)_{0},{ }^{7-11}$ and atomic axial tensors (AATs), $\mathbf{P}_{\dot{X}(m)}^{(a)}=\left(\partial \boldsymbol{m} / \partial \dot{\boldsymbol{X}}^{(a)}\right)_{0},{ }^{3-6,10-12}$ using eqns. (2a) and (2b), where $N$ is the number of atoms

$\left(\frac{\partial \boldsymbol{\mu}}{\partial Q_{t}}\right)_{0}=\sum_{a=1}^{N} \mathbf{P}_{X(\mu)}^{(a)} \boldsymbol{X}_{t}^{(a)}$

$$
\left(\frac{\partial \boldsymbol{m}}{\partial \dot{Q}_{t}}\right)_{0}=\sum_{a=1}^{N} \mathbf{P}_{\dot{X}(m)}^{(a)} \boldsymbol{X}_{t}^{(a)}
$$

and $\mathbf{X}_{t}^{(a)}$ is the atomic displacement vector of atom $a$ for normal mode $t$.

The APTs $\mathbf{P}^{a}$ and AATs $\mathbf{M}^{a}$ occurring in Stephens' formalism for $\mathrm{VCD}^{10.11}$ are related to those in eqn. (2) as in eqns. (3a) and (3b), where Im denotes the imaginary part

$\mathbf{P}_{X(\mu)}^{(a)}=\widetilde{\mathbf{P}}^{a}$

$\mathbf{P}_{\dot{X}(m)}^{(a)}=2 h \operatorname{Im} \widetilde{\mathbf{M}}^{a}$

( $\mathbf{M}^{a}$ is purely imaginary).

For the three normal rotational coordinates and the three normal translational coordinates eqn. (2a) can be expressed as in eqns. (4a) and (4b), respectively, familiar

$\left(\left(\boldsymbol{\mu}_{0}\right)\right) \equiv\left[\begin{array}{ccc}0 & \mu_{0 Z} & -\mu_{0 Y} \\ -\mu_{0 Z} & 0 & \mu_{0 X} \\ \mu_{0 Y} & -\mu_{0 X} & 0\end{array}\right]=\sum_{a=1}^{N} \mathbf{P}_{X(\mu)}^{(a)}\left(\left(\mathbf{Q}^{(a)}\right)\right)$

$\mathbf{0}_{3}=\sum_{a=1}^{N} \mathbf{P}_{X(\mu)}^{(a)}$ 
from the polar tensor IR intensity theory $;^{7,8}$ see also Refs. 9 and 13. Here, $\left(\left(\boldsymbol{\mu}_{0}\right)\right)$ represents $\boldsymbol{\mu}_{0}$ expressed as an antisymmetric second-rank axial tensor and $\left(\left(\varrho_{0}^{(a)}\right)\right)$ is defined analogously, where $\mathrm{e}_{0}^{(a)}$ is the equilibrium position vector of atom $a .0_{3}$ is a $3 \times 3$ zero matrix. The analogous rotational and translational mode equations provided by eqn. (2b) have been derived recently by Buckingham et al., ${ }^{4}$ as shown in eqns. (5a) and (5b); see also Ref. 6 for a derivation

$$
\begin{gathered}
\frac{1}{h} \mu_{\mathrm{N}} \boldsymbol{g}_{0} \mathbf{I}=\sum_{a=1}^{N} \mathbf{P}_{\dot{X}(m)}^{(a)}\left(\left(\mathbf{Q}_{0}^{(a)}\right)\right) \\
-\frac{1}{2 c}\left(\left(\boldsymbol{\mu}_{0}\right)\right)=\sum_{a=1}^{N} \mathbf{P}_{\dot{X}(m)}^{(a)}
\end{gathered}
$$

within the localized molecular orbital (LMO) model. Here, $\mu_{\mathrm{N}}$ is the nuclear magneton, $g_{0}$ is the molecular $g$-value tensor $^{14.15}$ and I is the moment of inertia tensor; see Ref. 13, in particular eqn. (34), for the corresponding equations in Stephens' formalism. The electronic contribution to the left-hand side of eqn. (5a) can be expressed in terms of the paramagnetic susceptibility, $\chi^{\mathrm{p}}$, of the molecule. ${ }^{4.16 .17}$ Analogous rotranslational sum rules occur in the formalism for nuclear shielding tensors ${ }^{18,19}$ from which the atomic tensors also can be obtained; ${ }^{4.17 .20 .21}$ see Ref. 13 for details.

In the polar tensor IR intensity theory Biarge et al. ${ }^{7}$ derived an intensity sum rule which takes into account the contributions from both the vibrational and the rotranslational modes. In their matrix formalism, and using the dipole strength $D_{t}$, this sum rule can be written as eqn. (6),

$\frac{h}{2} \sum_{a=1}^{N} \frac{1}{m_{a}} \operatorname{Tr}\left[\begin{array}{ll}\widetilde{\mathbf{P}}_{X(\mu)}^{(a)} & \mathbf{\mathbf { P }}_{X(\mu)}^{(a)}\end{array}\right]$

$=\sum_{t=1}^{3 N-6} \omega_{t} D_{t}+\frac{h}{2} \operatorname{Tr}\left[\left(\left(\tilde{\mu_{0}}\right)\right)\left(\left(\boldsymbol{\mu}_{0}\right)\right) \mathbf{I}^{-1}\right]$

where $\operatorname{Tr}$ signifies the trace of the matrix and $m_{a}$ is the mass of atom $a$; see also Refs. 8 and 22. The first, second and third terms will be denoted $\Sigma A_{l}(l=1$ to $3 N), \Sigma A_{l}(t=1$ to $3 N-6)$ and $\sum A_{\mathrm{R}_{\xi}}(\xi=X, Y, Z)$, respectively. $\Sigma A_{\mathrm{R}_{\xi}}$ is often referred to as the rotational correction.

Polavarapu was the first to apply an analogous approach to the intensities in VCD. ${ }^{5,23}$ However, the sum rule equation obtained was evaluated within the fixed partial charge (FPC) model, and the conclusions drawn were not quite general. The present author arrived at the same sum rule equation, which was evaluated ${ }^{6}$ within the LMO model. Taking into account the general appearances of the symmetry invariant atomic tensors, ${ }^{24}$ it was possible to formulate a general sum rule for $\Sigma R_{l}(l=1$ to $3 N)$ for chiral molecules. ${ }^{25}$ This sum was found to be zero only for certain molecules made chiral by isotopic substitution. On the basis of the work of Buckingham et al. ${ }^{4}$ a general expression can also be given for the rotational correction, $\sum R_{\mathrm{R} \xi}(\xi=X, Y, Z) .{ }^{25}$ The VCD intensity sum rule can be written as eqn. (7), where $\Sigma R_{l}$ and $\Sigma R_{\mathrm{R}_{\xi}}$ is the first and last

$\frac{h}{2} \sum_{a=1}^{N} \frac{1}{m_{a}} \operatorname{Tr}\left[\left|\mathbf{A}_{a}\right| \stackrel{\tilde{\mathbf{P}}}{X(\mu)}_{(a)} \ddot{\mathbf{P}}_{\dot{x^{\prime}(m)}}^{(a)}\right]$

$=\sum_{t=1}^{3 N-6} R_{t}+\frac{h}{2} \operatorname{Tr}\left[\left(\left(\tilde{\left.\boldsymbol{\mu}_{0}\right)}\right)\left(\frac{1}{h} \mu_{\mathrm{N}} \boldsymbol{g}_{0} \mathbf{I}\right) \mathbf{I}^{-1}\right]\right.$

term, respectively, and the various quantities are defined in eqns. (1b), (2), (4a) and (5a). The superscripts ${ }^{0}$ occurring in the left-hand sides of eqns. (6) and (7) indicate that the atomic tensors there are referred to their respective atomic coordinate systems, where they have their simplest appearances, ${ }^{24}$ and in eqn. (7) the determinant $\left|\boldsymbol{A}_{a}\right|$ of the $3 \times 3$ matrix transforming from the atomic coordinate system $a$ to the common coordinate system reflects the axial nature of the AAT. For clarity, the left-hand side expression from eqn. (5a) is given within parentheses in the last term of eqn. (7). If desired, that term can be simplified using the relationship I I ${ }^{-1}=\mathbf{E}_{3}$, a $3 \times 3$ unit matrix. ${ }^{25}$

It should be pointed out that the rotational corrections in eqns. (6) and (7) are to be evaluated in a centre-of-mass coordinate system, and as a result that should hold also for the other terms in these equations. However, these terms are origin-independent (for inexact wavefunctions the VCD terms may be origin-dependent; see below). Also note that, since a mere rotation does not influence the trace, it is not necessary to use the principal axes for the evaluation of the rotational corrections if another centreof-mass coordinate system happens to be more convenient.

Recently, Jalkanen et al. ${ }^{26.27}$ and Jalkanen et al. ${ }^{28}$ have performed extensive ab initio quantum-mechanical calculations of the atomic tensors and $D_{t}$ and $R_{t}$ of the chiral isotopomer of ammonia, NHTD, within Stephens' formalism $^{10.11}$ for VCD. This gives us an opportunity to apply the IR and VCD intensity sum rules. The application of the IR intensity sum rule is well-known, ${ }^{22.29}$ but is of interest here for a comparison. One reason for this is that most of the APTs in Ref. 28 do not fulfil eqn. (4b), i.e. for these atomic tensors the right-hand side of eqn. (4b) will deviate more or less from zero. As a result, the occurrence of translational corrections on the right-hand sides of eqns. (6) and (7) has to be considered. Since these intensity sum rules are based on linear transformations from derivatives with respect to the atomic displacement vectors, $\boldsymbol{X}^{(a)}\left(\right.$ or $\left.\dot{X}^{(a)}\right)$, on the left-hand sides to derivatives with respect to all the normal coordinates (or their time derivatives) on the right-hand sides (see the division of the atomic tensors into atomic vibration, rotation and translation tensors in Ref. 6), these translational corrections will take the form of eqns. (8) and (9). Here, $\widetilde{\mathbf{T}}=\left(T_{X}, T_{Y}, T_{Z}\right)$ are the three normal 
$\sum_{\xi=X, Y, Z} A_{T_{\xi}}=\frac{h}{2} \operatorname{Tr}\left[\left(\frac{\tilde{\partial \boldsymbol{\mu}}}{\partial \mathbf{T}}\right)_{0}\left(\frac{\partial \boldsymbol{\mu}}{\partial \mathbf{T}}\right)_{0}\right]=\frac{h}{2 m} \operatorname{Tr}\left[\tilde{\mathbf{0}_{3} \mathbf{0}_{3}}\right]$

$\sum_{\xi=X, Y, Z} R_{T \xi}=\frac{\hbar}{2} \operatorname{Tr}\left[\left(\frac{\tilde{\partial} \boldsymbol{\mu}}{\partial \mathbf{T}}\right)_{0}\left(\frac{\partial \boldsymbol{m}}{\partial \dot{\mathbf{T}}}\right)_{0}\right]$

$=\frac{h}{2 m} \operatorname{Tr}\left[\tilde{\mathbf{0}_{3}}\left(-\frac{1}{2 c}\left(\left(\boldsymbol{\mu}_{0}\right)\right)\right)\right]$

translational coordinates, and $\boldsymbol{0}_{3}$ and $-\frac{1}{2 c}\left(\left(\boldsymbol{\mu}_{0}\right)\right)$ are defined in eqns. (4b) and (5b), respectively. Since $\mathbf{0}_{3}$ is a zero tensor, the translational corrections are clearly zero and therefore not included in the original intensity sum rules. However, in the present application, all the quantities in the rotational and translational corrections are replaced by the corresponding right-hand side expressions from eqns. (4) and (5). With this approach the translational correction may be non-zero when eqn. (4b) is not fulfilled, as further elucidated below.

\section{Application of intensity sum rules to NHDT}

The atomic tensors in Refs. $26-28$ and 30 were calculated (A) with analytical derivative methods at the SCF level of approximation (operators $\mu$ and $\pi)^{26,27,30}$ and (B) via nuclear shielding tensors at the SCF-RPA level of approximation (operators R, P, F and L, K) ${ }^{28}$ Here $,{ }_{R}^{\mu},{ }_{P}^{\pi}$ and F refer to the use of electronic position, momentum and force operators, respectively, to obtain the APTs, and ${ }_{\mathrm{L}}^{\pi}$ and $\mathrm{K}$ refer to the use of electronic angular momentum and torque operators, respectively, to obtain the AATs. The fulfilment of the rotranslational sum rules by these atomic tensors of ammonia and some other molecules has recently been examined by Stephens et al. ${ }^{13}$ and, for ammonia, by the present author. ${ }^{31}$ In the latter work a modified rotranslational sum rule from eqn. (50) of Ref. 6 was used, relating the APTs and AATs. It has the advantage of being origin-independent for ammonia and its isotopomers even if eqn. (4b) is not fulfilled. It should be mentioned that Stephens et al. ${ }^{17}$ have also applied the rotational sum rule in eqn. (5a) expressed in terms of the paramagnetic susceptibility, $\chi^{p}$, and the electronic parts of the AATs, or the corresponding nuclear shielding tensors, to check the accuracy of the AATs of ethylene oxide. For the present application of intensity sum rules to ammonia it is of interest to know that only $\operatorname{APT}(\mu)^{26,27.30}$ fulfils eqn. (4b). ${ }^{13,31}$

For the calculation of the intensities ${ }^{26-28}$ the various APTs were applied in eqns. (2a) and (1a), yielding $D_{t}(\mathrm{~A}, \mathrm{~A})$ $\left(\mathrm{A}={ }_{\mathrm{R}}^{\mu}, \mathrm{P}, \mathrm{F}\right)$ for NHDT, and the various APTs and AATs were applied in eqns. (2) and (1b), yielding $R_{t}(\mathrm{DO}, \mathrm{A}, \mathrm{B}, \mathrm{C})$ $\left(\mathrm{A}, \mathrm{C}={ }_{\mathrm{R}}^{\mu},{ }_{\mathrm{P}}^{\pi}, \mathrm{F}\right)$ and $\left(\mathrm{B}={ }_{\mathrm{L}}^{\pi}, \mathrm{K}\right)$, where $\mathrm{A}, \mathrm{B}$ and $\mathrm{C}$ are the operators referred to above. [ $\operatorname{In} R_{t}(\mathrm{DO}, \mathrm{A}, \mathrm{B}, \mathrm{C})$ operator $\mathrm{A}$ characterizes the APT(A)s introduced via eqn. (2a) and operators B and C together define the AAT(DO,B,C)s introduced via eqn. (2b) according to the so-called distributed origin (DO) with origins at nuclei gauge. $]^{10,11,13} D_{t}$ is always origin-independent, while $R_{t}$, for inexact wavefunctions, is origin-independent only if $\mathrm{A}=\mathrm{C}$. ${ }^{28}$

The other terms in the intensity sum rule equations were calculated here via the right-hand sides of eqns. (4) and (5) from the various atomic tensors in Refs. 27, 28 and 30, and the resulting tensors were thereafter irtroduced into eqns. (6)-(9) for further evaluation. These evaluations, as well as the numerical calculations, were performed with the use of the program Theorist ${ }^{32}$ for Macintosh. The quantities $A$ and $R$ obtained were multiplied by $h e^{2} / \mathrm{amu}$ and $h e^{2} a_{0} / c \mathrm{amu},{ }^{33}$ respectively, for conversion from atomic units to Gaussian c.g.s. units. The evaluation of eqn. (9) showed that $\Sigma R_{T_{\xi}}=0$ by symmetry for ammonia and its isotopomers, i.e. independently of whether the right-hand side of eqn. (4b) is zero or not. According to the sum rule given in Ref. 25 it also holds that $\Sigma R_{l}=0$ for ammonia and its isotopomers. Thus for NHDT the VCD intensity sum rule in eqn. (7) is reduced to $\Sigma R_{t}=-\Sigma R_{R_{\xi}}$.

Applying the IR intensity sum rule, eqn. (6), to the tabulated $D_{t}(\mathrm{~A}, \mathrm{~A})$ values $\left(\mathrm{A}={ }_{\mathrm{R}}^{\mu}, \mathrm{P}, \mathrm{F}\right)$ and the corresponding APTs from Refs. 27 and 28 gives a fulfilment only for $D_{t}(\mu, \mu)$, as should be expected. However, with the translational correction from eqn. (8) included, eqn. (6) is also fulfilled for $D_{t}(\mathrm{~A}, \mathrm{~A})(\mathrm{A}=\mathrm{R}, \mathrm{P}, \mathrm{F})$. The most relevant terms are displayed in Table 1 for the various basis sets.

The tabulated origin-independent $R_{t}\left(\mathrm{DO},{ }_{\mathrm{R}}^{\mu}, \mathrm{A},{ }_{\mathrm{R}}^{\mu}\right)$ values $\left(\mathrm{A}={ }_{\mathrm{L}}^{\pi}, \mathrm{K}\right)$ and the corresponding atomic tensors from Refs. 27 and 28 were all found to fulfil the VCD intensity sum rule in eqn. (7), as may be seen from Table 1 .

\section{Results and discussion}

Considering first the IR intensities, we find from Table 1 that $\Sigma A_{t}(\mu, \mu)$ of data set (A) and the corresponding $\Sigma A_{t}(\mathrm{R}, \mathrm{R})$ of data set (B) differ for the smallest basis sets, and for these basis sets the translational correction of data (B), $\Sigma A_{T_{\xi}}(\mathrm{R}, \mathrm{R})$, is considerable; see in particular basis set $\mathrm{DZ}$ and note that three of the basis sets, DZ, DZ/1P and $\mathrm{TZ} / 2 \mathrm{P}$, are the same for data (A) and (B). Also note that $\Sigma A_{T_{\xi}}(\mu, \mu)$, not included in part (A) of Table 1, is zero for all basis sets, since $\operatorname{APT}(\mu)$ fulfils eqn. (4b). However, $\Sigma A_{t}(\mu, \mu)$ of the largest basis sets of data (A) is in good agreement with $\Sigma A_{t}(\mathrm{R}, \mathrm{R})$ of the largest conventional basis set, LZ95, and the largest polarized basis sets of data (B), and for these basis sets $\Sigma A_{T_{\xi}}(\mathrm{R}, \mathrm{R})$ is practically zero. $A_{i}(\mathrm{~A}, \mathrm{~A})(\mathrm{A}=\mathrm{P}, \mathrm{F})$ of data $(\mathrm{B})$ show larger deviations, and the translational corrections, $\Sigma A_{T_{\xi}}(\mathrm{A}, \mathrm{A})$, are small only for basis set LZ95, but about 10 (in the units of Table 1) for the largest polarized basis sets.

For the VCD intensities, we find that $\Sigma R_{t}(\mathrm{DO}, \mathrm{R}, \mathrm{L}, \mathrm{R})$ of data (B) is very large for the smallest conventional basis sets and varies much more with the basis sets than does the corresponding $\Sigma R_{t}(\mathrm{DO}, \mu, \pi, \mu)$ of data (A). However, for the largest polarized basis set of data (B), DZ/POL, there 
Table 1. Numerical values for NHDT ${ }^{a}$ of some relevant terms in the IR and VCD intensity sum rule eqns. (6) and (7). The translational correction required for the IR data (B) is defined in eqn. (8). The $A$ quantities are in $10^{-24} \mathrm{esu}^{2} \mathrm{~cm}^{2} \mathrm{~s}^{-1}, a^{2}$ the $R$ quantities in $10^{-44}$ $\mathrm{esu}^{2} \mathrm{~cm}^{2}$.

\begin{tabular}{|c|c|c|c|c|}
\hline \multicolumn{5}{|c|}{$(\mathrm{A})^{b}$ Data from Jalkanen et al. ${ }^{27}$} \\
\hline Basis $\operatorname{set}^{c}$ & $\Sigma A_{t}^{d}$ & $\left(\Sigma A_{l}-\Sigma A_{t}-\Sigma A_{R}\right)^{d}$ & $\Sigma R_{t}(\mathrm{DO})^{e}$ & $\sum R_{R_{\xi}}(\mathrm{DO})^{e}$ \\
\hline STO-3G & 7.49 & 0.01 & 0.086 & -0.085 \\
\hline $3-21 G$ & 13.51 & -0.02 & 0.136 & -0.136 \\
\hline 4-31G & 16.18 & 0.01 & 0.163 & -0.163 \\
\hline $6-31 G$ & 16.57 & 0.00 & 0.171 & -0.171 \\
\hline $\mathrm{DZ}$ & 17.67 & 0.01 & 0.203 & -0.293 \\
\hline $6-31 \mathrm{G}^{\star}$ & 13.47 & -0.02 & 0.096 & -0.006 \\
\hline $6-31 G^{\star \star}$ & 12.89 & 0.02 & 0.093 & -0.094 \\
\hline$D Z / 1 P$ & 13.83 & 0.01 & 0.114 & -0.115 \\
\hline $6-311 G^{\star \star}$ & 12.56 & -0.01 & 0.082 & -0.082 \\
\hline $6-31 G(E X T)$ & 11.72 & -0.01 & 0.076 & -0.077 \\
\hline$T Z / 2 P$ & 12.37 & 0.00 & 0.089 & -0.090 \\
\hline VD/3P & 11.37 & 0.02 & 0.074 & -0.074 \\
\hline
\end{tabular}

$(B)^{b}$ Data from Jalkanen et al. ${ }^{28}$

\begin{tabular}{|c|c|c|c|c|c|}
\hline Basis set ${ }^{c}$ & $\sum A_{t}^{f}$ & $\left(\Sigma A_{l}-\Sigma A_{t}-\Sigma A_{R_{\xi}}\right)^{f}$ & $\Sigma A_{T_{\xi}}{ }^{f}$ & $\sum R_{t}(\mathrm{DO})^{g}$ & $\sum R_{R_{\xi}}(\mathrm{DO})^{g}$ \\
\hline $\mathrm{DZ}$ & $\begin{array}{r}61.41 \\
74.29 \\
1019.63\end{array}$ & $\begin{array}{l}503.48 \\
664.65 \\
463.17\end{array}$ & $\begin{array}{l}503.47 \\
664.68 \\
463.26\end{array}$ & $\begin{array}{r}7.8 \\
12.1\end{array}$ & $\begin{array}{r}-7.721 \\
-12.190\end{array}$ \\
\hline $\mathrm{DZ} / 1 \mathrm{P}$ & $\begin{array}{r}10.73 \\
28.48 \\
558.55\end{array}$ & $\begin{array}{l}112.43 \\
243.94 \\
103.28\end{array}$ & $\begin{array}{l}112.47 \\
243.91 \\
103.47\end{array}$ & $\begin{array}{l}1.8 \\
2.7\end{array}$ & $\begin{array}{l}-1.963 \\
-2.689\end{array}$ \\
\hline LZ45 & $\begin{array}{r}7.03 \\
9.66 \\
20.89\end{array}$ & $\begin{array}{l}4.07 \\
6.92 \\
0.29\end{array}$ & $\begin{array}{l}4.09 \\
6.94 \\
0.28\end{array}$ & $\begin{array}{l}0.4 \\
0.4\end{array}$ & $\begin{array}{l}-0.361 \\
-0.452\end{array}$ \\
\hline$T Z / 2 P$ & $\begin{array}{r}7.87 \\
10.36 \\
49.27\end{array}$ & $\begin{array}{r}19.59 \\
27.39 \\
9.40\end{array}$ & $\begin{array}{r}19.60 \\
27.38 \\
9.43\end{array}$ & $\begin{array}{l}1.0 \\
1.1\end{array}$ & $\begin{array}{l}-0.960 \\
-1.072\end{array}$ \\
\hline LZ57 & $\begin{array}{r}6.16 \\
8.03 \\
13.36\end{array}$ & $\begin{array}{r}6.50 \\
10.22 \\
4.47\end{array}$ & $\begin{array}{r}6.52 \\
10.22 \\
4.50\end{array}$ & $\begin{array}{l}0.5 \\
0.6\end{array}$ & $\begin{array}{l}-0.452 \\
-0.502\end{array}$ \\
\hline LZ95 & $\begin{array}{l}11.79 \\
12.16 \\
13.46\end{array}$ & $\begin{array}{l}0.01 \\
0.03 \\
0.16\end{array}$ & $\begin{array}{l}0.00 \\
0.01 \\
0.19\end{array}$ & $\begin{array}{l}0.0 \\
0.1\end{array}$ & $\begin{array}{l}-0.040 \\
-0.034\end{array}$ \\
\hline VD/POL & $\begin{array}{r}4.33 \\
16.69 \\
12.68\end{array}$ & $\begin{array}{r}2.25 \\
118.25 \\
95.84\end{array}$ & $\begin{array}{r}2.27 \\
118.28 \\
95.83\end{array}$ & $\begin{array}{l}0.1 \\
0.0\end{array}$ & $\begin{array}{r}0.014 \\
-0.042\end{array}$ \\
\hline VD/DZ/POL & $\begin{array}{l}14.87 \\
12.80 \\
11.44\end{array}$ & $\begin{array}{r}0.06 \\
64.61 \\
63.04\end{array}$ & $\begin{array}{r}0.07 \\
64.63 \\
63.04\end{array}$ & $\begin{array}{l}0.1 \\
0.1\end{array}$ & $\begin{array}{l}-0.136 \\
-0.145\end{array}$ \\
\hline 6-31G/POL & $\begin{array}{l}11.67 \\
12.35 \\
12.54\end{array}$ & $\begin{array}{r}0.04 \\
9.47 \\
10.09\end{array}$ & $\begin{array}{r}0.03 \\
9.46 \\
10.11\end{array}$ & $\begin{array}{l}0.1 \\
0.1\end{array}$ & $\begin{array}{l}-0.142 \\
-0.150\end{array}$ \\
\hline DZ/POL & $\begin{array}{l}11.23 \\
12.72 \\
12.58\end{array}$ & $\begin{array}{r}-0.02 \\
14.40 \\
14.15\end{array}$ & $\begin{array}{r}0.02 \\
14.43 \\
14.13\end{array}$ & $\begin{array}{l}0.2 \\
0.0\end{array}$ & $\begin{array}{l}-0.065 \\
-0.066\end{array}$ \\
\hline
\end{tabular}

${ }^{a}$ Accurate atomic masses and coordinates of NHDT are given in Refs. 26 and 28 , respectively. ${ }^{b}$ The atomic tensors used have been calculated (A) with analytical derivative methods at the SCF level of approximation, ${ }^{27,30}$ and (B) via nuclear shielding tensors at the SCF-RPA level of approximation. ${ }^{28}$ cThe basis sets of the data sets $(A)$ and $(B)$ are defined in Tables 1 of Refs. 26 and 28 ,

respectively. ${ }^{d}$ The displayed quantities are $\Sigma A_{t}(\mu, \mu)$ (calculated from $\omega_{t}$ and $\left.D_{t}\right),{ }^{27} \Sigma A_{l}(\mu, \mu)$ and $\Sigma A_{R_{\xi}}(\mu, \mu)$, as defined in the text.

e The displayed quantities are $\Sigma R_{t}(\mathrm{DO}, \mu, \pi, \mu)$ (from $\left.R_{t}\right)^{27}$ and $\Sigma R_{R_{\xi}}(\mathrm{DO}, \mu, \pi, \mu)$, as defined in the text. ${ }^{f}$ The displayed quantities are $\sum A_{t}(\mathrm{~A}, \mathrm{~A})$ (calculated from $\omega_{t}$ and $\left.D_{t}\right),{ }^{28} \sum A_{t}(\mathrm{~A}, \mathrm{~A}), \Sigma A_{R \xi}(\mathrm{A}, \mathrm{A})$ and $\sum A_{T_{\xi}}(\mathrm{A}, \mathrm{A})$ with $\mathrm{A}=\mathrm{R}$ (first line), $\mathrm{P}$ (second line) and $\mathrm{F}$ (third line), as defined in the text. ${ }^{g}$ The displayed quantities are $R_{t}(D O, R, A, R)$ (from $\left.R_{t}\right)^{28}$ and $\Sigma R_{R_{\xi}}(D O, R, A, R)$ with $A=L$ (first line) and $K$ (second line), as defined in the text. 
is reasonable agreement with $\Sigma R_{t}$ of the largest data (A) basis sets [the low accuracy of the $\Sigma R$, values of data (B) is due to the low precision of $R_{t}$ given in Ref. 28; compare instead with $-\Sigma R_{R_{\xi}}$ from the last column]. For the smallest polarized basis set, VD/POL, the sign of $\Sigma R_{R_{\xi}}(\mathrm{DO}, \mathrm{R}, \mathrm{L}, \mathrm{R})$ [and probably also of $\Sigma R_{t}(\mathrm{DO}, \mathrm{R}, \mathrm{L}, \mathrm{R})$, if a higher precision had been used in Ref. 28] is opposite to that obtained with all the other basis sets. $\Sigma R_{t}(\mathrm{DO}, \mathrm{R}, \mathrm{K}, \mathrm{R})$ of data (B) is even larger for the smallest conventional basis sets, but for the other basis sets it shows behaviour similar to that of $\Sigma R_{t}(\mathrm{DO}, \mathrm{R}, \mathrm{L}, \mathrm{R})$; for a higher precision use instead $-\Sigma R_{R_{\xi}}(\mathrm{DO}, \mathrm{R}, \mathrm{K}, \mathrm{R})$ from the last column.

The IR translational correction, $\Sigma A_{T_{\xi}}$, gives in a single number a measure of the deviations from zero of the righthand side of eqn. (4b). (It is proportional to the sum of the squares of the elements of the resulting tensor.) However, even if $\Sigma A_{T_{\xi}}$ is zero, as for $\operatorname{APT}(\mu)$, fulfilment of the VCD intensity sum rule, eqn. (7), cannot be taken as evidence that the APTs and the corresponding AATs are mutually consistent. That can only be determined with the rotranslational sum rules [expressed here by eqns. (4a) and (5b)], as was done in Ref. 13, or with the use of eqn. (50) of Ref. 6, as was done in Ref. 31. In fact, $\operatorname{APT}(\mu)$ and $\operatorname{AAT}(D O, \pi, \mu)$ of ammonia are not consistent for the smallest basis sets. However, for the largest basis sets the deviations are small. It may therefore be concluded that the intensity sum rules, as applied here, are fulfilled even if the atomic tensors do not satisfy the rotranslational sum rules in eqns. (4) and (5). The reason is that all the terms in the intensity sum rules are calculated from the same set of atomic tensors, and that the atomic tensors used give origin-independent intensities. Thus, the usefulness of the intensity sum rules in this kind of application is rather that they offer convenient tests of the correctness of intensity calculations in IR and VCD for a given set of atomic tensors, and that they provide an accumulated effect of the basis set considered. For the largest basis sets there is reasonable agreement between results obtained with the two quantum-mechanical approaches, in particular when the electronic position operator, $\mu$ and $\mathrm{R}$ in the two approaches, is used for calculating the APTs.

Acknowledgements. I am indebted to Dr. Aatto Laaksonen for putting at my disposal a computer program for calculation of the inverse of the moment of inertia tensor and related quantities at the initial stage of this work.

\section{References}

1. Stephens, P. J. Croat. Chem. Acta 62(2B) (1989) 429.

2. Nafie, L. A. and Freedman, T. B. J. Phys. Chem. 90 (1986) 763.

3. Nafie, L. A. and Freedman, T. B. Chem. Phys. Lett. 134 (1987) 225.

4. Buckingham, A. D., Fowler, P. W. and Galwas, P. A. Chem. Phys. 112 (1987) 1.

5. Polavarapu, P. L. J. Chem. Phys. 86 (1987) 1136.

6. Rupprecht, A. Chem. Commun. Univ. Stockholm 7 (1987).

7. Biarge, J. F., Herranz, J. and Morcillo, J. An. R. Soc. Esp. Fis. Quim. A57 (1961) 81.

8. Person, W. B. and Newton, J. H. J. Chem. Phys. 61 (1974) 1040.

9. Fowler, P. W. and Buckingham, A. D. Chem. Phys. 98 (1985) 167.

10. Stephens, P. J. J. Phys. Chem. 91 (1987) 1712.

11. Stephens, P. J. J. Phys. Chem. 89 (1985) 748.

12. Rupprecht, A. Mol. Phys. 63 (1988) 951.

13. Stephens, P. J., Jalkanen, K. J., Amos, R. D., Lazzeretti, P. and Zanasi, R. J. Phys. Chem. 94 (1990) 1811.

14. Flygare, W. H. Chem. Rev. 74 (1974) 653.

15. Salzman, W. R. J. Phys. Chem. 93 (1989) 7351.

16. Stephens, P. J. and Jalkanen, K. J. J. Chem. Phys. 91 (1989) 1379.

17. Stephens, P. J., Jalkanen, K. J., Lazzeretti, P. and Zanasi, R. Chem. Phys. Lett. 156 (1989) 509.

18. Lazzeretti, P. and Zanasi, R. Phys. Rev. A 33 (1986) 3727.

19. Lazzeretti, P. and Zanasi, R. J. Chem. Phys. 87 (1987) 472.

20. Lazzeretti, P. and Zanasi, R. Chem. Phys. Lett. 112 (1984) 103.

21. Lazzeretti, P., Zanasi, R. and Stephens, P. J. J. Phys. Chem. 90 (1986) 6761.

22. King, W. T. In: Person, W. B. and Zerbi, G., Eds., Vibrational Intensities in Infrared and Raman Spectroscopy, Elsevier, Amsterdam 1982, p. 122.

23. Polavarapu, P. L. J. Chem. Phys. 84 (1986) 542.

24. Barron, L. D. Molecular Light Scattering and Optical Activity, Cambridge University Press, Cambridge 1982.

25. Rupprecht, A. Mol. Phys. 63 (1988) 955.

26. Jalkanen, K. J., Stephens, P. J., Amos, R. D. and Handy, N. C. Chem. Phys. Lett. 142 (1987) 153.

27. Jalkanen, K. J., Stephens, P. J., Amos, R. D. and Handy, N. C. J. Phys. Chem. 92 (1988) 1781.

28. Jalkanen, K. J., Stephens, P. J., Lazzeretti, P. and Zanasi, R. J. Chem. Phys. 90 (1989) 3204.

29. King, W. T. and Mast, G. B. J. Phys. Chem. 80 (1976) 2521.

30. Amos, R. D., Jalkanen, K. J. and Stephens, P. J. J. Phys. Chem. 92 (1988) 5571.

31. Rupprecht, A. Unpublished results.

32. Prescience Corporation, San Francisco, CA.

33. IUPAC Quantities, Units and Symbols in Physical Chemistry, Blackwell Scientific Publications, Oxford 1988.

Received May 24, 1990. 